\title{
Zip Code
}

National Cancer Institute

\section{Source}

National Cancer Institute. Zip Code. NCI Thesaurus. Code C25720.

A system designed to expedite the sorting and delivery of mail by assigning a series of numbers to each delivery area in the United States. Also used to refer to any individual delivery area code. 